\title{
EL ECOSISTEMA EMPRENDEDOR DE CÓRDOBA: UN ANÁLISIS SISTÉMICO
}

\section{The entrepreneurial ecosystem of Córdoba: a systemic analysis}

\section{Silvia Aisa', Daniel Parisi²}

Recibido: 16/09/2021 - Aceptado: 09/12/2021

\section{RESUMEN:}

La Provincia de Córdoba, Argentina, es reconocida nacional e internacionalmente por sus emprendimientos y sus instituciones de apoyo. Con el objetivo de cuantificar y dimensionar el ecosistema se realizó un relevamiento a instituciones de la provincia para analizar siete dimensiones: cultura emprendedora, sistema educativo, demanda local, capital social, entorno de los emprendedores, políticas públicas e infraestructura y RR.HH. del modelo elaborado por PRODEM y adaptado para este trabajo. Los resultados son diferenciados entre instituciones que están ubicadas en la ciudad capital de la provincia o en el interior, como así también el sector al que pertenecen.

\begin{abstract}
The Province of Córdoba, Argentina, is nationally and internationally acknowledged for its entrepreneurs and its support institutions. With the aim of quantify and size the entrepreneur ecosystem, a survey was carried out on institutions to analyze seven dimensions: entrepreneurial culture, educational system, local demand, social capital, environment, public policies and infrastructure and human resources of the model developed by PRODEM and adapted for this work. The results obtained are differentiated between institutions that are located in the capital city of the province or in the inland, as well as the sector they belong to.
\end{abstract}

PALABRAS CLAVE: emprendedores - ecosistema emprendedor - desarrollo económico emprendimiento

JEL: L26 - O38

KEYWORDS: entrepreneur - entrepreneurial ecosystem - economic development entrepeneurship

JEL: L26 - O38

\footnotetext{
${ }^{1}$ Profesora Facultad de Ciencias Económicas UNC. Córdoba. Argentina.silvia.aisa@gmail.com

2 Profesor Asistente. Facultad de Ciencias Económicas UNC. UES21. Córdoba. Argentina. danielparisi@unc.edu.ar
} 


\section{Introducción}

Los gobiernos han apostado a los emprendedores como un instrumento catalizador de desarrollo económico que, al fomentar la inversión, genera empleos y multiplica el bienestar. Sin embargo, en sí mismo, el gobierno no es determinante para que surja el emprendimiento. Se requiere de más elementos, actores e interacciones adecuadas para que puedan darse las condiciones óptimas que lo hagan posible. Aunque han existido diversos esfuerzos para impulsar actividades económicas, como clústeres, políticas industriales y el mismo fomento al emprendimiento, los emprendedores requieren algo más que un espacio físico y programas de apoyo y promoción. Para mantener a flote el desarrollo económico y consolidar la creación de nuevas empresas, los principales actores del ecosistema de emprendedores deberán interactuar entre sí y vincular sus estrategias.

Un ecosistema emprendedor se refiere a algo más que una cercanía física, ya que un emprendedor requiere formación, financiamiento y talento humano, así como un contexto cultural y social apropiado, además de un mercado abierto e infraestructura.

Cada ecosistema emprendedor tiene sus particularidades ya que dependen del entorno local en el que se desempeñan, de la historia de la región y la cultura de la sociedad. Sin embargo, al pensar en el emprendedor como parte de la estrategia de desarrollo de una región, éste se ubica en el centro de la dinámica económica.

El desarrollo de la comunidad emprendedora requiere un esfuerzo mancomunado: apoyo técnico y económico, fomento del espíritu emprendedor, trabajo conjunto entre el sector público y el privado y valorización de la actividad empresarial como motor del crecimiento.

Ciudades como San Francisco, Nueva York, Tel Aviv, Londres, Ámsterdam y Bangalore, han experimentado un importante crecimiento gracias a sus políticas y condiciones que han impulsado la innovación y el emprendimiento. Silicon Valley (California,
Estados Unidos) es en la actualidad uno de los ecosistemas de innovación y emprendimiento más fuertes y poderosos a nivel mundial. En el caso de Tel Aviv, posee un ecosistema con la experiencia y capacidad de producir emprendedores seriales, que crean, posicionan y venden empresas de alto valor. Londres es considerado el tercer ecosistema más importante del mundo, pues ha experimentado un importante crecimiento de sus industrias de base tecnológica, especializándose en Fintech. Bangalore, la quinta ciudad más poblada de la India, ha impulsado fuertemente empresas de base tecnológica (Bernal, 2019). América Latina, en comparación con otras regiones, tiene una alta propensión al emprendimiento, pero bajos resultados en innovación y en cantidad de emprendimientos de base tecnológica exitosos, ninguna empresa figura dentro de las mayores empresas globales de tecnología y las exportaciones de bienes y servicios digitales de la región son aún bajas.

La Ciudad de Córdoba, Argentina, es la segunda en orden de importancia del país, con una población de 1.3 millones de habitantes. Su ubicación estratégica en el corredor bioceánico y la presencia de universidades le permiten contar con recursos humanos calificados que le han permitido desarrollar un importante sector productivo. Esta diversificación productiva permitió desarrollar una red institucional que da apoyo y soporte al mundo emprendedor y empresarial que en los últimos años se fue fortaleciendo hasta alcanzar más de un centenar de organizaciones que contribuyen con el desarrollo al mundo de los negocios.

El objetivo del presente artículo es cuantificar y dimensionar el ecosistema emprendedor de Córdoba a través del análisis de siete dimensiones: cultura emprendedora, sistema educativo, demanda local, capital social, entorno de los emprendedores, políticas públicas e infraestructura y RR.HH . Para ello, se realizó un relevamiento a instituciones de apoyo emprendedor (incubadoras, aceleradoras, entre otras) de la provincia con el fin de calcular el puntaje de cada dimensión y clasificar al ecosistema emprendedor según 
su estadío: situación consolidada (puntaje de 7 a 10), intermedia (puntajes 5 y 6 ) y rezagado (puntajes 1 a 4)

Este artículo se organiza de la siguiente forma: en la primera sección se presentan conceptos y modelos teóricos sobre ecosistemas emprendedores. Luego, se muestra el perfil y composición de las instituciones de apoyo emprendedor relevadas. A continuación, se detalla el relevamiento llevado a cabo con el resultado de las percepciones o valoraciones, que hacen las instituciones del ecosistema emprendedor cordobés, sobre las siete dimensiones del enfoque sistémico. Finalmente, se discuten los hallazgos del trabajo y se sugieren los futuros abordajes de la temática.

\section{Conceptos y modelos teóricos sobre ecosistemas emprendedores}

\section{Los ecosistemas emprendedores: tipologías}

En el estudio de lo que debe entenderse por ecosistema del mundo emprendedor, el modelo más antiguo es el denominado modelo de la triple hélice. Se basa en entender el ecosistema emprendedor como la interacción entre los actores del sector privado, el gobierno, y la academia (Etzkowitz y Leydesdorff, 1995). En una segunda versión de este modelo se incorporó una cuarta hélice: la de la sociedad civil. Este modelo ha sido utilizado por décadas, principalmente aplicado a la generación de políticas públicas y posteriormente se comenzó a utilizar, y se continúa haciendo, para medir e impulsar la innovación abierta.

Este modelo fue cuestionado por resultar incompleto al momento de intentar profundizar y comprender las dinámicas complejas que suceden dentro de los ecosistemas modernos de emprendimiento, ya que se centran en una etiqueta sectorial más que en la función que cumplen dentro del ecosistema.

Para comprender en un sentido más amplio a los ecosistemas emprendedores se tornó útil utilizar las herramientas de mapeos, que permiten identificar los actores participantes con los cuales van a interactuar y generar si- nergias. Estos mapeos llevaron a una nueva concepción de ecosistemas: el modelo basado en actores que se compone de seis pilares y seis tipos de actores. Los seis pilares son:(1) Identificar, (2) capacitar, (3) conectar y sostener, (4) financiar, (5) habilitar y (6) celebrar, mientras que los seis tipos de actores son: (1) Articuladores, (2) Habilitadores, (3) Vinculadores, (4) Generadores de Conocimiento, (5) Promotores y (6) Comunidades. Para cada una de estas tipificaciones se asumen roles y valores, de ahí que suele conocerse también como modelo basado en actores, roles y valores.

En otra línea, los ecosistemas emprendedores están relacionados con los clústeres, sistemas regionales de innovación y redes. Bajo esta visión se identifican diez atributos culturales, sociales y materiales en los ecosistemas que son: cultura de apoyo, historias de emprendimiento y talento de los trabajadores, capital de inversión, redes, mentores y modelos a seguir, política y gobernanza, universidades, servicios de apoyo, infraestructura física y mercados abiertos (Spigel, 2017). Más allá de las visiones, está claro que el ecosistema emprendedor no se limita al conjunto de emprendedores, sino que incorpora una red más amplia e incluye actores como las instituciones de apoyo emprendedor, hasta valores y redes de la sociedad. Así, una de las características principales es que los ecosistemas emprendedores se han destacado por la capacidad que tienen de generar un tejido económico más competitivo e incrementar la riqueza y prosperidad de un área geográfica (García Cabrera, 2010).

En América Latina, el problema de la expansión del autoempleo informal en la región podría deberse, en parte, a la escasa creación de empresas dinámicas que sean capaces de generar empleo (Angelelli et al., 2006). Los gobiernos, habiendo reconocido el impacto que tienen en el desarrollo local y el empleo, han comenzado a impulsar los ecosistemas emprendedores e incluso han generado instituciones de carácter público como incubadoras y aceleradoras. En este marco, la creación de nuevas empresas puede fortalecerse a través de cinco características como son: políticas 
gubernamentales y procedimientos, condiciones socioeconómicas, habilidades emprendedoras y de negocios, apoyo financiero y asistencia no financiera (Marulanda, 2016).. En el mismo sentido, los factores claves para el fortalecimiento de un ecosistema son la diversidad de talento, minimizar barreras sociales, gran motivación, normas sociales que promuevan la velocidad, la colaboración y experimentación entre individuos (Hwang y Horowitt, 2012). Estos autores afirman que los ecosistemas son un sistema biológico y que el talento, las ideas y el capital son sus nutrientes. A su vez, coinciden en que existen diversas características y factores que conforman a un ecosistema de emprendimiento.

Finalmente, uno de los autores cuyas investigaciones han sobresalido en los últimos años es Daniel Isenberg quien desarrolló un diagrama en donde presenta los dominios que componen el ecosistema de emprendimiento: política, finanzas, cultura, soporte, capital humano y mercados (Isenberg, 2011).

Enfoque sistémico de los ecosistemas emprendedores

El modelo planteado por PRODEM se basa en siete dimensiones claves que inciden sobre la cantidad y calidad de las nuevas empresas que se crean. Permite detectar la incidencia de aquellos factores estructurales específicos particularmente importantes para los países en desarrollo, como los latinoamericanos (por ejemplo, condiciones sociales, estructura empresarial, capital social, cultura).

La primera de ellas es la existencia de emprendedores capaces de construir propuestas de valor potentes: el Capital Humano emprendedor. El proceso de surgimiento de estos emprendedores está influido por distintas cuestiones tales como, por ejemplo, los valores y creencias que conforman la Cultura, las Condiciones sociales de las familias donde las personas nacen y se forman y el funcionamiento del Sistema educativo. Más tarde en la adultez, las empresas en las que trabajan completarán una trayectoria de formación continua.
El enfoque sistémico también considera a aquellos factores que influyen sobre la existencia de oportunidades de negocios tales como las condiciones de la demanda, el tamaño y dinamismo del mercado, el perfil de las firmas que conforman la estructura empresarial y los esfuerzos registrados en el campo de la ciencia y tecnología, a los que el PRODEM denomina Plataforma de ciencia y tecnología para la innovación. La conversión de los proyectos en empresas, y su posterior desarrollo, dependen en buena medida de las capacidades de los emprendedores, pero es fundamental que ellos puedan acceder a una oferta apropiada de financiamiento para crear y hacer crecer el emprendimiento (para etapas tempranas, para expansión, capital de trabajo).

Otro factor clave es la existencia de Capital Social, es decir, de un ambiente de confianza que facilite la construcción de puentes para tejer redes de contacto con otros actores clave (empresarios, instituciones, etc.) y el acceso a recursos que, de otra manera, dificultarían la creación y el desarrollo del emprendimiento.

Por último, el proceso del emprendimiento se ve afectado por el contexto de Políticas y Regulaciones. Los gobiernos establecen distintas normas (por ejemplo, habilitaciones y permisos, impuestos y regulaciones al comercio exterior) que pueden resultarles más o menos amigables a los emprendedores. También implementan políticas que, por acción u omisión, acaban incidiendo sobre los emprendedores y sus empresas.

Así, el enfoque sistémico plantea que un ecosistema emprendedor consolidado debe tener desarrollado siete dimensiones: (1) Cultura emprendedora, (2) Sistema educativo, (3) Infraestructura y RR.HH., (4) Capital Social, (5) Demanda local, (6) Entorno de los emprendedores y (7) Políticas Públicas. Al fomentar estos aspectos, las condiciones resultan más favorables para que surjan nuevos emprendedores, se creen más y mejores empresas y que éstas logren desarrollarse y crecer. Las condiciones sistémicas para el emprendimiento en los diferentes países y 
su tasa de fertilidad empresarial, están positivamente correlacionadas. Por lo general, a mejores condiciones sistémicas le corresponden mayores tasas de creación de empresas, aunque no sea una relación lineal ni perfecta y esté sujeta a desfasajes temporales. Además, el nivel de condiciones sistémicas para el emprendimiento de los distintos países y su grado de desarrollo, también están altamente correlacionados. Los más desarrollados son los que tienen mejores condiciones para emprender y viceversa (Kantis, et al., 2012). Por lo tanto, dada la mayor capaci- dad de generar emprendimientos de calidad que tienen las naciones más avanzadas, es muy probable que las brechas de desarrollo tiendan a profundizarse de no mediar estrategias orientadas a mejorar las condiciones sistémicas del resto de los países.

Para caracterizar un ecosistema que abarque de manera óptima todas las dimensiones, las mismas deben ser valoradas con el máximo puntaje, por ejemplo 10 si la escala de cuantificación va de 1 a 10, según se muestra en la Figura 1 a continuación.

\section{Figura 1. Cuantificación óptima de las dimensiones de un ecosistema emprendedor.}

Fuente: Elaboración propia en base a PRODEM

\section{Perfil y composición de las instituciones de apoyo emprendedor.}

A los fines de desarrollar el trabajo, se conformó una base de datos de instituciones a partir de información provista por actores claves tales como Ministerio de Promoción del Empleo y de la Economía Familiar, la Facultad de Ciencias Económicas de la Universidad Nacional de Córdoba y el IERAL. El estudio se llevó a cabo a través de una encuesta a la cual respondieron el $81 \%$ del total resultando una cantidad de 101 instituciones de apoyo emprendedor de la Provincia de Córdoba.
En la Tabla 1 se muestra la información de la cantidad de instituciones que participaron del relevamiento, la cantidad de incubadoras y el empleo directo e indirecto generado distribuido según ubicación y sector.

En cuanto a la distribución geográfica, el 54\% de las instituciones relevadas están ubicadas en el interior provincial, mientras que el $46 \%$ restante se ubican en la Ciudad de Córdoba. En cuanto al sector al que pertenecen, el $40 \%$ son instituciones públicas y el $60 \%$ restante se encuadra en instituciones privadas, mixtas o académicas, que en adelante se las denominará "instituciones no públicas". 
Tabla 1. Perfil de las instituciones que acompañan a emprendedores de la Provincia de Córdoba

\begin{tabular}{lccccc}
\hline & Total & Capital & Interior & Pública & No pública \\
\hline Instituciones & 101 & 46 & 55 & 40 & 51 \\
Incubadoras & 30 & 19 & 11 & 6 & 24 \\
Empleo directo & 910 & 658 & 252 & 234 & 676 \\
Empleo indirecto & 1761 & 1382 & 379 & 275 & 1486
\end{tabular}

En cuanto al empleo que generan, se identificó que trabajan de forma directa en las instituciones unas 910 personas, la mayoría de ellas se encuentra en la capital cordobesa y en instituciones que no forman parte del sector público. Si se incluye el empleo indirecto que generan las instituciones, conocida como la red de la institución, incluyendo capacitadores, mentores, asesores contables o legales, coaches, entre otros, el número casi se duplica, precisamente alcanza 1.761 personas. De esta manera, el empleo directo e indirecto que generan las instituciones de apoyo emprendedor supera los 2.500 trabajadores.

Los servicios relevados que ofrecen las instituciones a los emprendedores, son los siguientes: capacitación y/o sensibilización (86\%), vinculación (67\%), financiamiento (34\%), incubación / aceleración (30\%) y otros como mentoreo, asesoramiento (para aplicar a programas de financiamiento, exportación, entre otros), espacio físico / coworking, difusión / visibilización / posicionamiento.

En cuanto a la forma jurídica de las instituciones relevadas, la principal figura elegida son fundaciones (24\%), luego asociaciones civiles (11\%) y tan solo el $9 \%$ son sociedades comerciales.

El público con el que trabajan las instituciones puede ser el emprendedor, en general (60\%), o bien un tipo de emprendedor específico (40\%). En este grupo, los servicios se especializan en alguna vertical o sector de los emprendimientos. En el relevamiento realizado sobresalen aquellas instituciones que apoyan únicamente a emprendedores tecnológicos (41\%), luego aquellas que se orientan a mujeres emprendedoras (25\%) y en tercer lugar las que se especializan en emprendimientos de triple impacto (22\%).

En cuanto a la propiedad del lugar donde las instituciones realizan sus operaciones, se encuentra que la mitad son dueños del espacio en el que trabajan y un $20 \%$ utiliza un espacio cedido por otra institución. Por otro lado, existe un $21 \%$ que alquila, mientras que existe un $7 \%$ de instituciones que no posee espacio, llevando a cabo sus operaciones de forma virtual o en espacios de terceros.

\section{Resultados de la cuantificación de las dimensiones del enfoque sistémico del ecosistema emprendedor de Córdoba}

\section{Resultados Generales}

En esta sección se presentan los resultados de la encuesta llevada a cabo a instituciones de apoyo emprendedor como incubadoras, aceleradoras, capacitadoras de emprendedores y toda aquella que tenga alguna acción o programa en fomento de emprendimientos de la Provincia de Córdoba en el mes de noviembre del año 2020.

Para dimensionar el ecosistema emprendedor de Córdoba se siguió el enfoque sistémico propuesto por Kantis et al. (2012), en el que se evalúan a través de preguntas de percepción que responden los referentes de las instituciones las siete dimensiones del enfoque. Cada una es evaluada en un puntaje del 1 al 10 y de su resultado resulta la siguiente clasificación: en situación consolidada (puntaje de 7 a 10), intermedia (puntajes 5 y 6 ) y rezagado (puntajes 1 a 4).

Las dimensiones que propone el enfoque sistémico para analizar un ecosistema emprendedor incluye: la cultura emprendedora, 
el sistema educativo, demanda local, capital social, infraestructura y RR.HH., entorno de los emprendedores y las políticas públicas. Para poder medirlas y cuantificarlas, los referentes de las instituciones respondieron una encuesta de 65 preguntas, separadas en 7 secciones, en la cual debían indicar el grado de acuerdo de una lista de afirmaciones con un puntaje del 1 al 10. Luego, para cada dimensión se calculó el promedio de los puntajes recibidos que se observan en el Figura 2.

Figura 2. Cuantificación de las dimensiones sistémicas del ecosistema emprendedor de Córdoba (unidades en puntos en escala del 1 al 10)

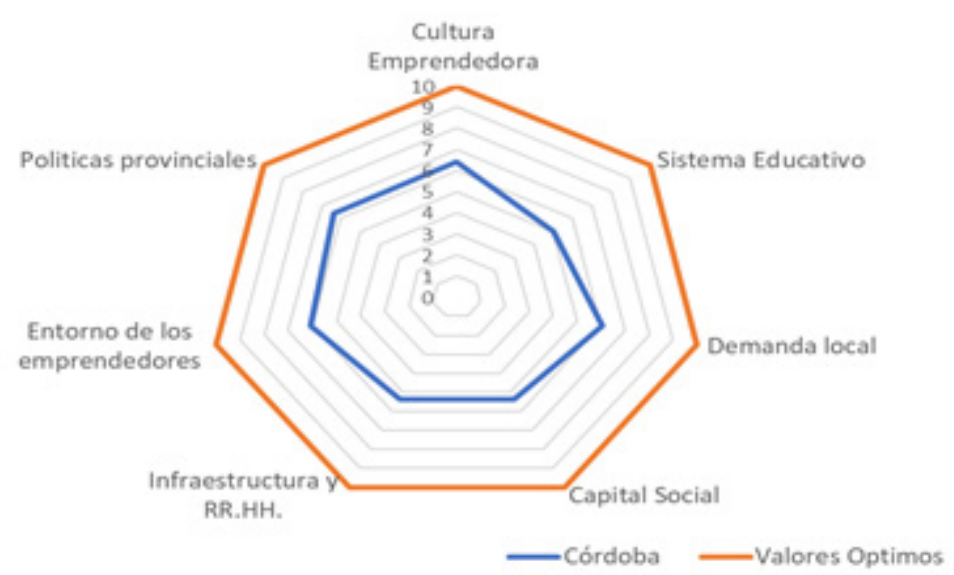

En la Figura 2 se muestran los resultados promedio de cada una de las dimensiones analizadas. Se observa que la cuantificación más alta en el territorio cordobés es el de la cultura emprendedora, (con un puntaje promedio de 6,42); en segundo lugar, el de las políticas provinciales (con un puntaje de 6,37), y en tercer lugar el entorno emprendedor (puntaje 6,20). Cabe aclarar que ninguna dimensión superó el puntaje de 7 , que indica una alta valoración y conformidad con la realidad, lo que se denomina un ecosistema emprendedor consolidado. Las dimensiones menos valoradas por las instituciones locales son el capital social, la infraestructura y RR.HH. y el sistema educativo.

En la Tabla 2 se detallan los valores de toda la muestra, observándose una gran concentración de puntaje entre los valores 5 y 6, lo cual da una idea que la realidad emprendedora de Córdoba resulta de un nivel intermedio. El aná-

\section{Tabla 2. Dimensiones sistémicas del ecosistema emprendedor de Córdoba} (unidades en puntos en escala del 1 al 10)

\begin{tabular}{lccccc}
\hline Dimensión & Total & Capital & Interior & Pública & No pública \\
\hline Cultura Emprendedora & 6,42 & 6,90 & 6,01 & 6,20 & 6,60 \\
Sistema Educativo & 5,03 & 5,20 & 4,89 & 4,90 & 5,20 \\
Demanda local & 6,03 & 6,95 & 5,21 & 5,40 & 6,40 \\
Capital Social & 5,37 & 5,38 & 5,38 & 5,10 & 5,60 \\
Infraestructura y RR.HH. & 5,35 & 5,68 & 5,05 & 5,50 & 5,30 \\
Entorno de los emprendedores & 6,09 & 6,94 & 5,56 & 6,00 & 6,40 \\
Políticas Provinciales & 6,37 & 6,57 & 5,81 & 6,40 & 6,00
\end{tabular}


lisis se realiza distinguiendo entre instituciones que se ubican en la ciudad capital de Córdoba (capital) con aquellas que se ubican en el interior provincial (interior). También se analiza las instituciones por sector público (públicas) que son todos los organismos del gobierno provincial y/o municipal con programas y acciones de apoyo del emprendedor y las instituciones de otros sectores (no públicas) que pueden ser: privadas, académicas (como las incubadoras de las universidades) o mixtas.

Realizando un análisis por tipo de instituciones, se observa una diferencia entre las de capital con las del interior. Los puntajes promedios de las primeras son mayores en todas las dimensiones que las segundas, por lo que se puede concluir que el ecosistema emprendedor de la Ciudad de Córdoba está más consolidado que el del interior. En el análisis por sector, son las instituciones "no públicas" quienes perciben mejor las dimensiones, salvo en la de políticas provinciales, en el que las instituciones públicas lo valoran más positivamente.

\section{Resultados por Dimensión}

Para profundizar el análisis de los resultados anteriormente expuestos, se analiza en esta sección cada dimensión, desagregándolas en los puntos que incluye cada una, de manera que permita identificar los fuertes y débiles al interior de cada una de ellas.

\section{- Dimensión Cultura Emprendedora}

Se entiende por cultura emprendedora al conjunto de valores y creencias que contribuyen a que el emprendimiento sea una alternativa ocupacional deseable (por ejemplo, las actitudes frente al riesgo y al fracaso, la presencia de modelos de rol y pymes destacadas locales) así como también el de las instituciones educativas, que pueden desarrollar las competencias para emprender. Las preguntas que se incluyeron en la encuesta para medir esta dimensión fueron:

1. ¿En qué medida, en su ciudad los empresarios locales son reconocidos y valorados por la sociedad?

2. ¿En qué medida, en su ciudad la gente considera a la opción emprendedora como una alternativa ocupacional relevante?

3. ¿En qué medida, en su ciudad el fracaso se considera una fuente de aprendizaje y no se lo sanciona socialmente?

4. ¿En qué medida, en su ciudad existen emprendedores/as y empresarios/as destacados/as que sirven como modelos de rol (figuras inspiradoras)?

5. ¿En qué medida, en su ciudad los medios de comunicación locales relevantes difunden casos de emprendedores y empresas jóvenes exitosas y se interesan por el tema?

En la Tabla 3 se observan los resultados para cada una de las preguntas anteriores. El aspecto más valorado sobre la cultura emprendedora en Córdoba es que existen emprendedores/empresarios modelos que inspiran a otros (puntaje de 7,12 ) y casi con un puntaje similar la cuantificación sobre la opción emprendedora como una alternativa de vida (puntaje 7,10). En contraste, los factores menos valorados abordan sobre la difusión en medios locales de casos de emprendedores (puntaje de 5,94 ) y que el fracaso no es sancionado socialmente y es una fuente de aprendizaje (puntaje de 5,94).

\section{Tabla 3. Cuantificación sobre la Cultura Emprendedora}

(unidades en puntos en escala del 1 al 10)

\begin{tabular}{lccccc}
\hline ¿En qué medida, en su ciudad... & Total & Capital & Interior & Pública & No pública \\
\hline 1.Empresarios reconocidos y valorados & 6,76 & 7,04 & 6,49 & 6,40 & 7,00 \\
2.Opción de vida "emprender" & 7,10 & 7,42 & 6,83 & 7,10 & 7,10 \\
3.Fracaso no es sancionado & 5,22 & 5,38 & 5,09 & 5,40 & 5,10 \\
4.Emprendedores modelos que inspiran & 7,12 & 8,00 & 6,34 & 6,70 & 7,40 \\
5.Temática emprend. en los medios & 5,94 & 6,66 & 5,28 & 5,40 & 6,30
\end{tabular}


Las instituciones de la capital cordobesa resaltan aún más la presencia de emprendedores/ empresarios modelos, ya que la valoración promedio de este punto alcanza el 8. En el interior este punto se posiciona en tercer lugar con un puntaje de 6,34 siendo el primero, para este grupo de instituciones, el de emprender como una opción de vida. Ambos grupos de instituciones ubicaron al punto de que el fracaso como fuente de aprendizaje en la última posición Cabe aclarar que todos los puntos de la dimensión cultura emprendedora tienen un promedio mayor para Ciudad de Córdoba que para el interior. En la distinción por sector, los resultados no son tan claros aunque en la mayoría de los puntos las valoraciones promedios son mayores para las instituciones que no forman parte del sector público.

\section{- Dimensión Educación}

En la dimensión de Educación se analiza la opinión de las instituciones sobre la educación formal que atraviesan los jóvenes en los cursos del secundario y superior en cuanto a contenidos que sean útiles para el mundo emprendedor. Las preguntas que se incluyeron en la encuesta para medir esta dimensión fueron:

1. ¿En qué medida, en su ciudad los institutos terciarios y universidades tienen cursos o programas orientados al desarrollo de las capacidades emprendedoras de sus estudiantes

2. ¿En qué medida, en su ciudad los cursos o programas realizados por los institutos terciarios y universidades contribuyen de una manera significativa al desarrollo de las capacidades emprendedoras de sus estudiantes?

3. ¿En qué medida, en su ciudad estos cursos o programas realizados por los institutos terciarios y universidades alcanzan a una porción mayoritaria de los estudiantes de ese nivel?

4. ¿En qué medida, en su ciudad las escuelas secundarias tienen cursos o actividades educativas orientadas al desarrollo de las capacidades emprendedoras de sus estudiantes? 5. ¿En qué medida, en su ciudad los cursos o actividades educativas realizadas por las escuelas secundarias contribuyen de una manera significativa al desarrollo de las capacidades emprendedoras de sus estudiantes?

6. ¿En qué medida, en su ciudad estos cursos o actividades educativas realizadas por las escuelas secundarias alcanzan a una porción mayoritaria de los estudiantes de ese nivel?

En la Tabla 4 se analiza el resultado de cada una de ellas diferenciando entre educación superior, que incluye estudios terciarios y universitarios, con los de la educación secundaria. Entre los resultados resalta que la educación superior como formadora de potenciales emprendedores es mejor valorada que la educación secundaria, con puntajes de 5,43 contra 4,64 respectivamente. Sin embargo, en el interior (puntaje de 5,07 para la educación secundaria y 4,72 para la educación superior) y para las instituciones públicas (puntaje de 5,07 para la educación secundaria y 4,72 para la educación superior) ocurre lo contrario

Tabla 4. Cuantificación sobre la Educación (unidades en puntos en escala del 1 al 10)

\begin{tabular}{lccccc}
\hline ¿En qué medida, en su ciudad... & Puntaje & Capital & Interior & Pública & No pública \\
\hline $\begin{array}{l}\text { Educación superior } \\
\begin{array}{l}\text { 1. Disponibilidad de cursos y } \\
\quad \text { programas }\end{array}\end{array} \quad 5,43$ & 6,51 & 4,72 & 4,53 & 6,10 \\
$\begin{array}{l}\text { 2. Contribución al desarrollo } \\
\quad \text { emprendedor }\end{array}$ & 5,70 & 6,56 & 4,87 & 4,40 & 6,50 \\
$\begin{array}{l}\text { 3. Alcance de los cursos sobre } \\
\text { emprendedor }\end{array}$ & 4,94 & 5,70 & 4,26 & 4,10 & 5,50 \\
\hline $\begin{array}{l}\text { Educación secundaria } \\
\text { 4. Disponibilidad de cursos y programas }\end{array}$ & 4,64 & 4,15 & 5,07 & 5,33 & 4,23 \\
$\begin{array}{l}\text { 5. Contribución al desarrollo } \\
\text { emprendedor }\end{array}$ & 4,72 & 4,30 & 5,21 & 5,60 & 4,30 \\
$\begin{array}{l}\text { 6. Alcance de los cursos sobre } \\
\text { emprendedor }\end{array}$ & 4,42 & 4,08 & 4,72 & 5,00 & 4,10
\end{tabular}


Las instituciones de capital en los tres puntos de cada nivel que incluye la dimensión consideran mejor a la educación superior que la secundaria como herramienta formadora de emprendedores, incluso en algunos la diferencia en los valores se aproxima a los 2 puntos. Ocurre lo contrario con las instituciones del interior en el que valoran más la educación secundaria que la superior.

En cuanto al análisis por sector de la institución, la educación superior es más valorada en las instituciones no públicas mientras que la educación secundaria lo es para las instituciones públicas.

En lo que respecta al punto sobre la disponibilidad de cursos y programas que ofrece la educación superior y secundaria nuevamente se observa el mismo patrón. La primera (educación superior) es mejor vista que la segunda (educación secundaria) para el total de respuesta aunque para las del interior y las públicas ocurre lo contrario.

El aspecto menos valorado es el alcance de los cursos de formación emprendedora que ofrece el sistema educativo (puntaje de 4,42) y esta posición se obtiene en todos los subgrupos analizados.

Si bien existe esta diferencia entre la educación secundaria y superior los puntajes que esta dimensión obtiene son bajos, llegando en algunos casos a apenas superar el puntaje de 4. Por ello podemos decir que las instituciones del ecosistema emprendedor de Córdoba perciben que la educación formal está rezagada en gran partes de los temas relacionados al mundo emprendedor.

- Dimensión Demanda local

Se entiende por Demanda local al espacio de oportunidades locales formado por las condiciones de mercado, empresas, proveedores, clientes, entidades y otros dinamizadores. Las preguntas que se incluyeron en la encuesta para medir esta dimensión fueron:

1. ¿En qué medida, en su ciudad las actividades económicas principales (y sus cadenas de valor) generan nuevas oportunidades y demandas que pueden ser aprovechadas por los emprendedores y empresas jóvenes locales?

2. ¿En qué medida, en su ciudad se realizan ferias o encuentros donde participan empresarios de otras provincias, regiones o países? 3. ¿En qué medida, en su ciudad existen proyectos y/o empresas nuevas y jóvenes con potencial de crecimiento basados en ideas o tendencias observadas en otras ciudades, regiones o países?

En la Tabla 5 se encuentran las valoraciones promedios para cada una de estas preguntas. El punto más fuerte de esta dimensión es el las oportunidades de mercado locales (puntaje de 6,30) mientras que el punto más débil la existencia de feria donde participan emprendedores de otros lugares (puntaje de 5,60 ). Analizando por cada una de las categorías, se observa que la participación en ferias locales está más consolidada en la Ciudad Capital que en el interior. Por el lado del sector de la institución, son las que no forman parte del sector público la que mejor valoran la demanda local de Córdoba.

\section{Tabla 5. Cuantificación sobre la Demanda local}

(unidades en puntos en escala del 1 al 10)

\begin{tabular}{lccccc}
\hline ¿En qué medida, en su ciudad... & Puntaje & Capital & Interior & Pública & No pública \\
\hline 1. Hay oportunidades de mercado & 6,30 & 6,76 & 5,89 & 6,20 & 6,40 \\
$\begin{array}{l}\text { 2. Participación de emprendimientos } \\
\text { de otros lugares en ferias locales }\end{array}$ & 5,60 & 6,80 & 4,53 & 4,90 & 6,10 \\
$\begin{array}{l}\text { 3. Emprendimientos basados en } \\
\text { ideas de otros lugares }\end{array}$ & 6,20 & 7,30 & 5,21 & 5,20 & 6,90 \\
& & & & &
\end{tabular}


- Dimensión Capital Social

La dimensión Capital Social, que representa el conjunto de redes entre emprendedores, entre emprendedores e instituciones formales e informales, como así también a la facilidad para acceder a las mismas es analizada en la Tabla 6. Las preguntas que se incluyeron en la encuesta para medir esta dimensión fueron:

1. ¿En qué medida, en su ciudad es común que la gente confíe en las personas que no forman parte de su círculo más cercano?

2. ¿En qué medida, en su ciudad es común que las personas se relacionen entre sí sin importar los segmentos sociales a los que pertenecen?
A continuación, en la Tabla 6, se muestran las valoraciones de estas preguntas para el total de respuestas y para los subgrupos de instituciones que se vienen analizando. Como puede verse, la confianza con personas que no forman parte de su círculo para realizar proyectos en conjunto o bien establecer redes obtuvo un puntaje de 5,50 siendo superior para las instituciones del interior (puntaje de 5,55) que de la capital (puntaje 5,46) mientras que el punto sobre si las personas se relacionan entre sí sin importar el segmento social obtuvo un puntaje de 5,24 siendo superior para el grupo de instituciones de la Capital (puntaje de 5,30 ) y que no forman parte del sector público (puntaje de 5,40).

\section{Tabla 6. Cuantificación sobre la Dimensión Capital Social}

(unidades en puntos en escala del 1 al 10)

\begin{tabular}{lccccc}
\hline ¿En qué medida, en su ciudad... & Puntaje & Capital & Interior & Pública & No pública \\
\hline $\begin{array}{c}\text { 1. Confianza en personas que no } \\
\text { forman parte del círculo cercano }\end{array}$ & 5,50 & 5,46 & 5,55 & 5,20 & 5,70 \\
$\begin{array}{c}\text { 2. Interrelación entre personas de } \\
5,24\end{array}$ & 5,30 & 5,21 & 5,00 & 5,40
\end{tabular}

Dado que los valores son todos superiores a 5 el capital social del ecosistema emprendedor está en una situación intermedia aunque sus valores están muy cerca del límite inferior de esta clasificación.

\section{- Dimensión Infraestructura y RRHH}

La dimensión Infraestructura y RR.HH incluye los factores que promueven la creación y el desarrollo de los emprendimientos como la tecnología, servicios disponibles, el transporte y la distribución y la presencia de personas idóneas para incorporar al emprendimiento. Las preguntas que se incluyeron en la encuesta para medir esta dimensión fueron:

1. ¿En qué medida, en su ciudad conseguir y retener recursos humanos de calidad es un obstáculo para las empresas jóvenes y proyectos de emprendimiento?

2. ¿En qué medida, en su ciudad el acceso a telecomunicaciones e internet de calidad es un obstáculo para las empresas jóvenes y proyectos de emprendimiento? 3. ¿En qué medida, en su ciudad el acceso a servicios de logística, transporte y rutas adecuados es un obstáculo para las empresas jóvenes y proyectos de emprendimiento?

4. ¿En qué medida, en su ciudad el acceso a servicios públicos (agua, electricidad, gas) adecuados es un obstáculo para las empresas jóvenes y proyectos de emprendimiento?

Según las respuestas de las instituciones a estas preguntas, el principal obstáculo que tiene un emprendedor es el de conseguir y retener recursos humanos de calidad (puntaje de 6,76 ) siendo la valoración superior en las de capital (puntaje de 7,20 ) que en las del interior (puntaje de 6,34). Luego, el acceso a internet, servicios de logísticas y servicios públicos no son valorados como un obstáculo importante para el ecosistema emprendedor dado que sus puntajes apenas superan los 5 puntos, salvo en la Ciudad de Córdoba que la valoración de los servicios de transporte alcanzó el 5,82. En la Tabla 7 se observan estos resultados. 
Tabla 7. Cuantificación sobre Infraestructura y de RR.HH.

(unidades en puntos en escala del 1 al 10)

\section{¿En qué medida, en su ciudad} existen los obstáculos...

Total Capital Interior Pública No pública

1. Conseguir y retener RR.HH idóneos

$\begin{array}{lllll}6,76 & 7,20 & 6,34 & 6,80 & 6,80 \\ 4,94 & 5,04 & 4,83 & 5,30 & 4,70\end{array}$

2. Acceso a telecomunicaciones e 4,9 internet de calidad

3. Acceso a servicios de logística, transporte y rutas adecuados

$\begin{array}{lllll}5,20 & 5,82 & 4,64 & 5,10 & 5,30\end{array}$

4. Acceso a servicios públicos (agua,

- Dimensión Entorno Emprendedor

El entorno de los emprendedores incluye los apoyos que la localidad ofrece a los emprendedores para fortalecer y crecer en sus proyectos. Las preguntas que se incluyeron en la encuesta para medir esta dimensión fueron:

1. ¿En qué medida los apoyos de actores locales (empresarios, sector público e instituciones) contribuyen efectivamente a capacitar y entrenar a los emprendedores y empresarios jóvenes para el desarrollo de sus negocios?

2. ¿En qué medida estos apoyos contribuyen efectivamente a acompañar y asistir a los emprendedores y empresarios jóvenes en el planteo y validación de sus modelos de negocios?

3. ¿En qué medida estos apoyos contribuyen efectivamente a vincular a los emprendedores y empresarios jóvenes con programas públicos de apoyo (provinciales o nacionales)?
4. ¿En qué medida estos apoyos contribuyen efectivamente a vincular a los emprendedores y empresarios jóvenes con fuentes de financiamiento privadas?

5. ¿En qué medida estos apoyos contribuyen efectivamente a vincular a los emprendedores y empresarios jóvenes con potenciales clientes y proveedores?

6. ¿En qué medida estos apoyos contribuyen efectivamente a ayudar a que las empresas nuevas y jóvenes crezcan?

En la tabla 8 se muestran cada una de las valoraciones a estas preguntas. El mayor puntaje para las instituciones fue el punto que los apoyos contribuyen a capacitar y entrenar a emprendedores y empresarios jóvenes (puntaje 6,66). Luego, con poca diferencia, se ubica el punto de que los apoyos contribuyen a vincular a los emprendedores con programas públicos de apoyo (puntaje de 6,58).

Tabla 8. Cuantificación sobre el entorno de los emprendedores (unidades en puntos en escala del 1 al 10)

\section{¿En qué medida, en su ciudad los} apoyos locales (empresas, gobierno e instituciones) contribuyen...

1. A capacitar y entrenar a los Total Capital Interior Pública No pública emprendedores

2. Al planteo y validación de modelos de negocios de emprendedores

3. A vincular a los emprendedores con programas públicos de apoyo

4. A vincular a los emprend. con fuentes de financ. privadas

5. A vincular a los emprendedores con potenciales clientes y proveedores

6. efectivamente a ayudar a que los emprendimientos crezcan

$\begin{array}{lllll}6,66 & 7,58 & 5,81 & 6,40 & 6,90 \\ 6,30 & 7,42 & 5,28 & 5,90 & 6,60 \\ 6,58 & 6,96 & 6,23 & 6,70 & 6,50 \\ 5,06 & 5,62 & 4,57 & 5,00 & 5,10 \\ 5,84 & 6,46 & 5,28 & 5,60 & 6,00 \\ 6,12 & 6,92 & 5,40 & 5,90 & 6,30\end{array}$


En contraposición, el punto con menos puntaje fue el de que los apoyos contribuyen a vincular con fuentes de financiamiento privado (puntaje de 5,06) y también el de los apoyos permiten vínculos con clientes y proveedores (puntaje de 5,84) ambos con puntajes menores a 6 puntos.

Los resultados muestran que el entorno emprendedor es más valorado en la Capital que en el interior provincial ya que todas sus valoraciones son más altas. A su vez las instituciones públicas valoraron con menor puntaje esta dimensión que las instituciones que no forman parte del gobierno, en la mayoría de los puntos.

\section{- Dimensión Políticas Públicas}

Por último, en cuanto a las políticas públicas, los resultados se muestran en la Tabla 9. Las preguntas que se incluyeron en la encuesta para medir esta dimensión fueron:

1. ¿En qué medida el apoyo a los emprendedores con potencial y a las pymes jóvenes está presente en la agenda de políticas y acciones de la provincia?
2. ¿En qué medida existen programas públicos provinciales que brindan apoyo no financiero (p.ej. capacitación, tutorías, asesoramiento, etc.) para aquellos emprendedores con potencial y pymes jóvenes?

3. ¿En qué medida existen programas públicos provinciales que financian a emprendedores con potencial del crecimiento (p.ej. capital semilla)?.

4. ¿En qué medida existen programas públicos provinciales de financiamiento para pymes jóvenes en etapa de crecimiento?

Los programas de apoyo no financiero del gobierno (provincial y municipal) son considerados una fortaleza para las instituciones del ecosistema emprendedor ya que, salvo las del interior, sus valoraciones superan los 7 puntos. En contraposición, los programas de financiamiento tanto para emprendedores como para Pymes jóvenes fueron valorados con puntajes alrededor de los 6 puntos. Las instituciones del interior fueron las que peor puntuaron esta dimensión. En la tabla 9, se observan todos los resultados de esta dimensión.

\section{Tabla 9. Cuantificación sobre políticas públicas}

(unidades en puntos en escala del 1 al 10)

\begin{tabular}{lccccc}
\hline ¿En qué medida, en su ciudad... & Puntaje & Capital & Interior & Pública & No pública \\
\hline $\begin{array}{l}\text { 1. El apoyo a emprendedores está } \\
\text { presente en la agenda pública }\end{array}$ & 6,98 & 7,62 & 6,38 & 6,80 & 7,10 \\
$\begin{array}{l}\text { 2. Existen programas que brindan } \\
\quad \text { apoyo no financiero }\end{array}$ & 7,02 & 7,54 & 6,57 & 7,10 & 7,00 \\
$\begin{array}{l}\text { 3. Existen programas públicos que } \\
\quad \text { inancian a emprendedores }\end{array}$ & 5,94 & 6,30 & 5,62 & 6,20 & 5,80 \\
4. Existen programas públicos de & 5,54 & 5,88 & 5,25 & 6,00 & 5,30
\end{tabular}

\section{Conclusión}

Los ecosistemas emprendedores conforman un conjunto de actores que contribuye a mejorar las condiciones de contexto dentro de las cuales se desarrolla el emprendimiento. El acceso a fuentes de financiamiento, personal capacitado, entramado institucional y las redes que puede desarrollar son algunos de los principales factores que resultan relevantes tanto al momento de creación de un emprendimiento como en sus primeros años de vida. La Provincia de Córdoba es una de las referentes del país en el tema emprendedor debido a la existencia de un gran número de incubadoras, aceleradoras e instituciones de formación emprendedora que ofrecen un conjunto de servicios para el crecimiento y consolidación de los emprendedores.

En este trabajo se analizaron los resultados 
de un relevamiento de más de 100 instituciones de apoyo emprendedor de la Provincia de Córdoba, las cuales valoraron distintos aspectos que conforman el ecosistema emprendedor provincial. La mayoría de éstas ofrecen servicios relacionados a la formación emprendedora como actividades de capacitación y sensibilización. Otro punto destacado son sus acciones de vinculación y networking que llevan a cabo y que resultan muy valoradas por los emprendedores.

Las instituciones valoraron siete dimensiones del enfoque sistémico propuesto por Kantis et al. (2012) que sirven para dimensionar, cuantificar y analizar el funcionamiento del ecosistema emprendedor de Córdoba.

Los hallazgos principales fueron que el ecosistema emprendedor de Córdoba, valorado por las instituciones que lo conforman, se encuentra en un estadío intermedio (dado que, el promedio de cada una de las siete dimensiones, se encuentran en un rango entre 5 y 6 en una escala de 1 a 10 puntos). Los mejores puntajes surgieron en la cultura emprendedora $(6,42)$, entendiéndola como aquellos valores y creencias que contribuyen a que emprender sea una opción de vida, y el entorno emprendedor $(6,09)$, aquella red de vínculos y de apoyo. Entre las dimensiones valoradas con menor puntaje están: el sistema educativo como formador de emprendedores, el capital social y la infraestructura y RR.HH, siendo estas dimensiones las que debieran promoverse en futuras políticas públicas o acciones que encaren las instituciones educativas o incluso el propio sector emprendedor.

Sin embargo, entrando en detalles en las partes que componen a cada dimensión, existen algunos aspectos que se encuentran consolidados (aquellos con puntaje promedio superior a 7), como la existencia de emprendedores modelos que inspiran, que la idea de que emprender es una opción de vida o que existen suficientes programas de apoyo no financieros. En el otro extremo, los aspectos más rezagados se encuentran relacionados con la educación formal (secundaria y universitaria) como formadora de emprende- dores con puntajes promedios inferiores a 5 .

Analizando subgrupos de las instituciones, se encontró que el ecosistema emprendedor de la Ciudad de Córdoba (Capital) está en mejor posición que el del interior provincial, dado que todas las dimensiones estuvieron mejor valoradas para el primer grupo. En cuanto a las instituciones del sector público que conforman el ecosistema emprendedor, sus valoraciones sobre las dimensiones analizadas son menores que las del sector privado y académico. Esto significa que el sector que no forma parte del sector público tiene una mejor perspectiva del funcionamiento del ecosistema emprendedor de Córdoba.

Dado que el ecosistema emprendedor se compone principalmente de instituciones y de emprendedores, es importante avanzar en el estudio sobre las valoraciones que hagan éstos de las dimensiones. A su vez, es importante replicarlo en el tiempo, así como también poder llevarlo a cabo en otras provincias que permita su comparación.

\section{REFERENCIA BIBLIOGRAFICAS}

- Angelelli, P., Moury, R. y Llisterri, J. J. (2006). "Analysis of National Capacities to Support Small Enterprises in Latin America". (Sustainable Development Department, NSN N 136). Washington D.C.: Banco Interamericano de Desarrollo.

- Banco Interamericano de Desarrollo -BID(2018) “Doing Bussiness. Full Report”Ed. 15, Washington DC.

- CAF (2013) “Emprendimientos en América Latina: Desde la subsistencia hacia la transformación productiva". Disponible en: publicaciones.caf.com Bogotá, Colombia.

- Engel, J. S. (2015). Global Clusters of Innovation: Lessons from Silicon Valley. California Management Review, 57(2), 36-65.

- Etzkowitz H., Leydesdorff L. (1995).The Triple Helix - University -Industry-Government Relations: A Laboratory for Knowledge Based Economic Development. Glycoconjugate Journal January 1995 14(1):14-9

- García Cabrera, A. M., García Soto, M. G. (2010). Ecosistema emprendedor para las empresas de base tecnologica. TEC empre- 
sarial, 4(1), 8-21.

- Global Entrepreneurship Monitor - GEM (2013). Monitor Global de la Actividad Emprendedora. Informe 2013. Disponible en: https://www.cise.es/wp-content/uploads/ GEM-2013-Global-Report.pdf

- Hwang, V., \& Horowitt, G. (2012). The rainforest. Los Altos Hills, California: Regenwald.

- Isenberg, D. (2011). The entrepreneur ecosystem strategy as a new paradigm for economic policy: Principles for cultivating entrepreneurship. Babson Global, s/n.

- Kantis, H., Federico, J. y Menéndez, C. (2012). "Políticas de fomento al emprendimiento dinámico: Tendencias y desafíos". Documento de Trabajo CAF N²012/09. Caracas: CAF

- Kantis H.; Federico, J.; Ibarra García S. et al. (2016) “Condiciones sistémicas para el emprendimiento dinámico 2016: novedades y tendencias para fortalecer e integrar los ecosistemas de la región" $1^{\circ}$ ed . - Rafaela : Asociación Civil Red Pymes Mercosur, 2016.

- Kantis H.; Federico J.; Ibarra García S. (2015) "Ciudades para emprender" documento de Presidencia de la Nación. Ministerio de Producción y Trabajo de la Nación. Buenos Aires, Argentina.

- Marulanda Valencia, F. A. (2016). Entorno y motivaciones para emprender. Revista Escuela de Administración y Negocios (EAN), 60(81), 12 - 28.

- Mejía, J. (2017). El modelo de Israel start-up. Nueva Revista, (143), 29 -36.

- Sánchez, Y., García, F., \& Mendoza, J. (2015). La capacidad de innovación y su relación con el emprendimiento en las regiones de México. Estudios Gerenciales, 31(136), 243-252. 